\title{
GOBERNANZA DE LOS MACRODATOS Y DEMOCRACIA REPRESENTATIVA
}

Marco Betzu, Giovanni Coinu, Gianmario Demuro 
SUMARIO

1. MACRODATOS Y DEMOCRACIA. 2. LA MULTIFUNCIONALIDAD DE LOS MACRODATOS. 3. MACRODATOS, POPULISMO Y DEMOCRACIA REPRESENTATIVA. 


\title{
GOBERNANZA DE LOS MACRODATOS Y DEMOCRACIA REPRESENTATIVA
}

\author{
MARCO BETZU, GIOVANNI COINU, GIANMARIO DEMURO' \\ Universidad de Cagliari, Italia
}

\section{MACRODATOS Y DEMOCRACIA.}

La globalización y la difusión de la Red determinan, entre otros muchos efectos, el de la pérdida del territorio por parte del regulador estatal. El fenómeno de la evanescencia de un espacio cerrado de la regulación tiene muchas analogías con la demanda creciente de una regulación que sea capaz de mantener el control estatal y que, al mismo tiempo, pueda expresarse también más allá de las fronteras nacionales. En efecto, si el soberano ya no tiene un territorio que gobernar, ¿cómo podrá mantenerse como tal soberano, y seguir pudiendo contar con extender su influencia más allá de sus fronteras? Históricamente también la cultura, aun en su aspiración universal, tiene una dimensión territorial o, mejor, una identidad cultural tiene un arraigo precisamente dentro de confines determinados.

Y por último la democracia, también con vocación universal, está ligada a la dimensión territorial y está, en consecuencia, plasmada diversamente en los distintos contextos territoriales. El territorio está también aquí absolutamente presente y, en cada ocasión, atrae hacia sí la gravedad de las decisiones regulatorias. Como si la democracia quisiera expandirse desde sus vínculos territoriales, pero cada vez se viese obligada a retroceder al interior de sus confines para poder recuperar la capacidad regulatoria.

${ }^{1}$ El párrafo 1 debe atribuirse a G. Demuro, el párrafo 2 a G. Coinu, el párrafo 3 a M. Betzu. Texto basado en la Ponencia presentada en el XIV Congreso Iberoamericano de Derecho Constitucional, 21 - 23 de mayo de 2019, Universidad De Buenos Aires.

El presente trabajo delimita el campo de investigación del proyecto "Governance dei big data e democrazia rappresentativa" financiado en 2019 por Regione Sardegna en virtud de la ley regional n. 7/2007.

Gianmario Demuro. Professore Ordinario (Email: demuro@unica.it). Marco Betzu. Professore Associato (Email: mbetzu@unica.it). Giovanni Coinu. Professore Associato (Email: gcoinu@unica.it). Dipartimento di Giurisprudenza. Università di Cagliari. Via Sant'Ignazio, 17 - 09123 Cagliari. 
El retorno a los confines territoriales también puede ser una aspiración de las distintas versiones de retorno al populismo en las democracias occidentales, según el paradigma estudiado por Mounk, que identifica la propuesta populista en la voluntad de hacer coincidir al pueblo con la nación que se contrapone a la democracia representativa, la cual, en cambio, sería la base de la expansión de élites enfrentadas a la voluntad popular ${ }^{2}$.

En este contexto de crisis de la idea de democracia representativa se expande la tecnología que, de por sí, tiende a gobernar los factores de producción en ausencia de vínculos territoriales.

El soberano sin territorio se debe ocupar de tecnología y tiene solamente el derecho para gobernarla, y para el derecho constitucional la innovación digital se convierte en el «campo» de prueba para medir la efectividad de los instrumentos que ha utilizado en contextos preexistentes.

El tema que nos proponemos profundizar es el de la gobernanza de los macrodatos (big data), fenómeno que está a la base de toda la llamada economía circular ${ }^{3}$ y que tiene una serie de consecuencias sobre la idea misma de representación política.

\section{LA MULTIFUNCIONALIDAD DE LOS MACRODATOS.}

¿Puede la inteligencia artificial influir en los resultados de una consulta democrática? ¿O ser utilizados como soporte del responsable político? ¿Y es posible prever instrumentos normativos capaces de disciplinar la gestión de los macrodatos en la elaboración, ejecución y evaluación de las políticas públicas?

Los macrodatos, sobre la base de sus principales características, pueden ser definidos en general mediante la regla de las « $3 \mathrm{~V} »$ : volumen, variedad, velocidad ${ }^{4}$.

El Volumen está ligado a la cantidad y a la calidad de los datos generados a raíz del crecimiento de las actividades on-line, del uso de smartphone y plataformas en Internet: una enorme cantidad de datos heterogéneos que pueden referirse a edad, sexo, localización geográfica, perfil demográfico, composición familiar, hábitos alimentarios, datos biométricos, preferencias comerciales, o capacidad de gasto de las personas.

La Velocidad, en cambio, reside en la rapidez con que estos volúmenes se adquieren y elaboran; de ello derivan en primer lugar las cuestiones referidas a la actualidad del dato y a su actualización.

La Variedad, por último, es debida a las innumerables fuentes de las cuales se obtienen los datos: páginas HTML en cualquier lengua, tuits, sensores, detectores técnicos, científicos, fotografías, documentos, datos de geolocalización.

${ }^{2}$ Y. Mounk, The People vs. Democracy, Harvard University Press, Cambridge Massachussetts, 2018, passim

3 J. Rifkin, The Third Industrial Revolution, Palgrave Macmillan, New York, 2011, passim.

${ }^{4}$ M. A. Beyer - D. Laney, The Importance of "Big Data": A Definition, Gartner report, 2012. 
Las $3 \mathrm{~V}$ determinan la necesidad de elaborar los datos a través de algoritmos matemáticos capaces de poner en evidencia correlaciones no detectables con la utilización de instrumentos tradicionales de análisis y detección. El valor de las informaciones extraíbles no es intrínseco, sino que reside en la capacidad de organizarlas, analizarlas y medirlas, para obtener luego factores y decisiones.

Por una parte, comprender las potencialidades de la capacidad predictiva de los macrodatos y, por otra, verificar su legítimo aprovechamiento para influir en los procesos democráticos y las decisiones en las políticas públicas, constituye un reto fascinante pero todavía no afrontado cabalmente por la doctrina jurídica a nivel internacional.

La Unión Europea define los macrodatos como «una gran cantidad de diferentes tipos de datos producidos a alta velocidad a partir de un gran número de diversos tipos de fuentes» (COM (2014) 442 Final). La gestión de estos dataset, de elevada variabilidad y en tiempo real, impone el recurso a nuevos instrumentos y métodos, como por ejemplo potentes procesadores, software y algoritmos. Los métodos tradicionales de análisis, normalmente, producen correlaciones lineales, en términos de relación causa-efecto, y utilizan métodos de muestreo que implican la elección previa de los aspectos a estudiar, de los datos que deben seleccionarse para comprender un determinado fenómeno. Los datos «tradicionales» (smalldata) se han elaborado habitualmente para analizar fenómenos a través de la construcción de una hipótesis, la elección de variables e indicadores con el fin de identificar causas y posibles efectos.

Los macrodatos, al contrario, han determinado una variación sustancial de la estructura del método de análisis, que cambia por la extensión y el impacto que estas informaciones son capaces de generar en la sociedad. El objetivo de la elaboración de los macrodatos, pues, no es (solo) explicar los fenómenos, sino sobre todo evidenciar correlaciones entre variables que serían indetectables con los instrumentos de análisis tradicionales.

Se llega así al concepto de elaboración de los macrodatos, definida como «la capacidad de recoger detallada y continuamente todos los datos necesarios para la reconstrucción de hechos y fenómenos, junto a la de elaborar todos los datos de forma que produzcan información, conocimiento completo, original e incluso predictivo sobre los fenómenos» ${ }^{5}$. La capacidad predictiva, de naturaleza probabilística, es un rasgo típico del empleo de los macrodatos, pues actuando sobre una gran mole de datos los resultados de la elaboración se acercan de forma muy fiable a lo que podría suceder.

El llamado data mining, es decir el conjunto de técnicas y metodologías orientadas a la extracción de información de grandes cantidades de datos, plantea pues importantes cuestiones en el plano jurídico. Se acusa con frecuencia a estas operaciones de ser realizadas en forma poco transparente. Del tratamiento de estos datos, como es previsible, se origina un conflicto irreductible entre garantía de confidencialidad, por

5 M. FAlCone, Big Data e pubblica amministrazione: nuove prospettive per la funzione conoscitiva pubblica, en Rivistra trimestrale di diritto pubblico, 4/2017, p. 622. 
un lado, y derecho de acceso a la información, por otro. Fricción inevitable, desde el momento en que privacy y macrodatos «no aparecen en relieve como entidades absolutas, sino como bienes-intereses relativos, es decir, cada uno comprimibile para permitir un razonable espacio de vida al otro según cuanto exige la norma constitucional de la coexistencia de valores plurales y antagonistas» ${ }^{6}$. Tal delicado equilibrio caracteriza, pues, cualquier uso que se haga de esta ingente cantidad de datos, en el campo tanto público como privado.

Sin embargo, las cuestiones de interés jurídico ya no están limitadas, como ocurría hasta ahora, a la mera peligrosidad social referida a la violación de las libertades individuales. El tratamiento de los macrodatos permite, de hecho, la consecución de macrofinalidades a través de actividades de recogida y tratamiento de datos en forma agregada que descubren relaciones entre datos, normas, hábitos y tendencias ${ }^{7}$.

El relieve asumido por los macrodatos en el ámbito económico, para aumentar la eficacia de los procesos productivos, para mejorar la capacidad de toma de decisiones de los administradores y, sobre todo, para interpretar mejor las tendencias de mercado, diversificando la publicidad y propuestas comerciales, es de conocimiento común. Y efectivamente han sido objeto de la disciplina antitrust, a protección de la libertad de competencia entre las empresas y represión de las prácticas desleales.

Los macrodatos adquieren relieve también con respecto al contexto público e institucional. Es posible identificar dos macrodimensiones de incidencia de estos datos: una dimensión negativa, cuando los datos se configuran como instrumentos de conservación o de salvaguardia de un statu quo; y una dimensión positiva, cuando los datos se utilizan como instrumento orientado a la progresión material de la sociedad, en el ámbito político, económico y social.

Entra sin duda en la primera dimensión el uso de los datos como medida para la lucha contra el terrorismo. Como se recordará, a raíz de los atentados terroristas de Madrid (2004) y Londres (2005), fue aprobada la directiva 2006/24/CE, llamada Data retention, sobre la conservación de los datos personales de tráfico telefónico y telemático. Esta imponía, en particular, la conservación de todos los datos relativos al tráfico relacionado con la telefonía fija, la móvil, el acceso a Internet y el correo electrónico, así como la telefonía por Internet. Se trataba, en otros términos, de una medida de prevención del fenómeno terrorista que afrontaba todos los principales medios de comunicación. Sin embargo, esta medida fue considerada excesivamente erosiva del principio de protección de la privacy y de la protección de datos personales, sancionados respectivamente por los artículos 7 y 8 de la Carta de los Derechos Fundamentales de la Unión Europea. Con la sentencia de 8 de abril de 2014 (asuntos acumulados C-293/12 y C-594/12, Digital Rights Ireland Ltd contra Irlanda), en efecto, la Corte de Justicia estimó que la directiva implicaba una evidente injerencia en los derechos

${ }^{6}$ M. F. De Tullio, La privacy e i big data verso una dimensione costituzionale collettiva, en Politica del diritto, 4/2016, p. 639.

7 Este interesante tema se desarrollará durante la investigación de la cual este trabajo representa la primera comparación con la comunidad científica internacional. 
fundamentales de la casi totalidad de la población europea. Más concretamente, de la parte motiva de la sentencia emerge cómo la vigilancia a gran escala no puede ser generalizada e indiscriminada, sino que puede producirse solo en correlación con una específica sospecha de delito, a raíz de un mandato judicial y por indicación ex lege de las autoridades competentes, de los procedimientos y condiciones que se deben observar. La directiva Data retention, por el contrario, aparecía en contraste con los principios enunciados en la Carta de los Derechos de la Unión Europea, pues el período de conservación de datos previsto para fines de protección del orden público resultaba excesivo y no proporcional, y también por la poca claridad de las condiciones a las que dicha conservación estaba subordinada.

En definitiva, pues, el uso de los macrodatos en su dimensión negativa, como instrumento destinado a la prevención del fenómeno terrorista, y por tanto la conservación de un estatus, encuentra un límite infranqueable en el respeto de un núcleo mínimo, pero irreductible, del derecho a la intimidad y a la protección de datos personales.

En lo referente a la dimensión positiva, es decir esa dimensión por la que los datos tienen como finalidad principal el progreso, incluso material, de la sociedad, es posible distinguir dos campos de acción.

El primero, más tradicional y ya objeto de atención de los estudiosos, se refiere al empleo de los macrodatos por parte de algunos organismos públicos y en la fase instructora de determinados procedimientos administrativos. La Agencia Tributaria, por ejemplo, ha empezado a utilizar los macrodatos en el ámbito del derecho tributario; el Istituto nazionale italiano di Statistica (ISTAT), por su parte, en la elaboración del Programa Estadístico Nacional 2014-2016; el Istituto Nazionale italiano della Previdenza Sociale (INPS) y el Istituto nazionale italiano per l'Assicurazione contro gli Infortuni sul Lavoro (INAIL), por último, han adoptado políticas de gestión de datos, dotándose de medios tecnológicos adecuados a las exigencias de datification de los macrodatos, elaborándolos, con carácter experimental, en apoyo de los propios procesos decisorios. Por lo que respecta, en cambio, al uso de los macrodatos en apoyo de la acción administrativa, las administraciones públicas, en aras del principio de verdad material, están comenzando a utilizar los datos para integrar su propia actividad instructora. Aunque este uso muestre ciertos límites — baste pensar, por ejemplo, en la no idoneidad de la disciplina actual sobre datos públicos para ser aplicada a la lógica de los macrodatos-, se trata de una innovación de alcance histórico, capaz de incidir, modificándola a la raíz, en la fase de instrucción del procedimiento administrativo. Por otra parte, es subyacente al principio de buena administración, según el art. 97 Const., el hecho de que la administración pública recoja, archive y reutilice los datos en su poder para garantizar una mejor relación entre los resultados alcanzados y los objetivos fijados.

El segundo campo, en cambio, menos explorado, se refiere a la posibilidad de que los datos puedan ser utilizados al servicio del responsable de decisión político, como instrumento para la elaboración de políticas públicas que sean más adherentes a las 
preferencias de los ciudadanos. En otros términos, en una fase de fuerte deslegitimación de los canales tradicionales de representación política, se trata de evaluar si el uso de los datos en apoyo del responsable político puede representar un instrumento alternativo, pero no sustitutivo, para la elaboración de políticas públicas más adecuadas para satisfacer las necesidades de los ciudadanos. En caso afirmativo, habrá luego que identificar los instrumentos normativos capaces de regular la incidencia de los datos sobre las distintas fases del ciclo de actuación política ${ }^{8}$.

No obstante, se trata en este caso de entender en qué modo los datos ya en poder de las administraciones públicas, o en todo caso recuperables a través de canales institucionales, pueden ser conscientemente utilizados para la elaboración de políticas públicas que respondan lo más posible a las preferencias del ciudadano.

Sobre este punto, asumen un papel de primer plano los open data, es decir, aquellos datos no sujetos a restricciones particulares, libremente accesibles y reutilizables. Su utilización tiene, en efecto, una doble proyección positiva: en primer lugar, permite al responsable político basar sus decisiones en datos de los cuales emerge, bien delineado, el contexto sobre el que luego incidirán las políticas; en segundo lugar, permite al ciudadano comprobar las razones que han llevado al responsable político a preferir una política a otra.

Un sistema de este tipo, basado en la máxima valorización de los macrodatos, encuentra, por otra parte, acomodo tanto a nivel europeo como a nivel estatal.

Por el lado europeo, la Agenda digital para Europa se propone ayudar a los ciudadanos y las empresas a maximizar sus beneficios a través de las tecnologías digitales, contemplando entre sus objetivos el desarrollo de los macrodatos como instrumento para generar crecimiento económico. Concepto reiterado también en el documento «A European strategy on the data value chain», donde se evidencia cómo las cadenas de datos son el núcleo de la futura economía del conocimiento. Los datos generados por sistemas interoperables, en efecto, pueden ser utilizados para llegar a mejores decisiones, capaz de inducir desarrollo económico. Por ejemplo, en los servicios públicos: es evidente que su mayor eficiencia pasa por la compartición de datos entre un sector público y otro, lo que permite, además, una mejor percepción de conjunto de los servicios prestados. Interacción que, sin embargo, no puede limitarse solo al ámbito público. Según el citado documento, en efecto, para maximizar la contribución de los open data, es necesario el desarrollo de un sistema formado no solo por organismos públicos, entes de investigación e instituciones académicas, sino también por pequeñas y medianas empresas y por grandes sociedades TIC que cuenten con las competencias para valorizar este gran patrimonio informativo. Usados de este modo, los open data pueden determinar tangibles efectos económicos, tanto respecto al mercado como respecto al perfil laboral. A este propósito, si para 2016 se preveía un mercado directo de los open data del valor de 55.300 millones de euros, se estima que, en 2020, las

${ }^{8}$ Este tema se desarrollará durante la investigación de la cual este trabajo representa la primera comparación con la comunidad científica internacional. 
dimensiones del mismo aumentarán en un 36,9\%, alcanzando así un valor de 75.700 millones. En cuanto al crecimiento del empleo, mientras que para 2016 se estimaba un incremento de 75.000 puestos de trabajo, en el año 2020 esta cifra se situará en torno a las 100.000 unidades (Creating Vaine through Open Fecha). Se estima, finalmente, también para el año 2020, un crecimiento del PIB, para los 28 países miembros, de 250.000 millones de euros, gracias a la implantación del Data Driven Decision Making, es decir, el sistema de elaboración de las decisiones basadas en datos procedentes de sistemas interconectados en red.

El uso de los datos como instrumento de apoyo del responsable político asume cada vez mayor relieve también a nivel estatal. En las Líneas guía para la valorización del patrimonio informativo público (2015 - Agenzia per l'Italia digitale) se evidencia, efectivamente, que los datos en posesión de la Administración pública, a diferencia del pasado, en el que tenían un papel funcional únicamente para el logro de las tareas institucionales, ahora adquieren un diferente significado en términos de estímulo al desarrollo de la economía digital. Los macrodatos representan, por tanto, un patrimonio al que corresponde un valor económico real, que debe ser necesariamente considerado como motor para el desarrollo del mercado, para el nacimiento de nuevas figuras profesionales y para el apoyo a las decisiones de todo responsable político, incluso local.

El uso de los macrodatos puede ser igualmente valioso, por último, en la fase de evaluación de las políticas públicas. En efecto, en cuanto la intervención encuentre su fundamento, al menos parcial, en los datos, será posible analizar cuáles han sido los resultados de la misma y qué factores los han determinado.

En definitiva, pues, el uso de los datos como soporte del proceso de toma de decisiones puede incidir favorablemente en todas las fases del ciclo de actuación política.

\section{MACRODATOS, POPULISMO Y DEMOCRACIA REPRESENTATIVA.}

Más en general —y el tema toca la relación entre evolución digital y crisis de la democracia representativa - es cierto en cambio que los macrodatos inciden significativamente en el ámbito político-institucional.

Una prueba de ello son las últimas elecciones presidenciales estadounidenses. Al respecto, en una entrevista concedida a La Stampa, Alexander Nix, consejero delegado de Cambridge Analytica, declaró: «Creo que el modo de hacer campañas electorales está cambiando. [...] Antes se hacía campaña electoral encomendándose a sondeos de opinión y a la intuición de los candidatos y sus equipos. Hoy tenemos la posibilidad de utilizar grandes cantidades de datos que nos permiten crear modelos de análisis predictivo de las inclinaciones y del comportamiento. Con esta información podemos identificar y alcanzar con mensajes eficaces a los electores: sabemos cuáles son los 
temas de interés y por lo tanto hablamos de lo que importa a los votantes, dando matices diferentes a la comunicación para garantizar el éxito de la misma»?.

La campaña electoral que permitió a Trump ganar las últimas elecciones presidenciales representa, pues, un buen ejemplo que muestra cómo el uso de los datos está modificando la relación entre ciudadanos e instituciones. Este fenómeno, sin embargo, no se limita solo al contexto americano, sino que se está extendiendo poco a poco al europeo, donde no es inusual que las decisiones se basen también sobre investigaciones de mercado, o datos obtenibles principalmente de actores privados.

Se ha evidenciado que «la introducción de los macrodatos en el arsenal del estratega político puede permitir un control pormenorizado de la organización de las campañas electorales, desde la planificación hasta la definición de la agenda, la construcción del consenso y el seguimiento de los resultados» ${ }^{10}$. En particular, el trabajo apenas citado ha demostrado cómo, a partir de la elaboración de perfiles de los electores, es posible determinar «la disponibilidad de los mismos a participar en las actividades de campaña» (behaviour); «prever las preferencias políticas y electorales de los ciudadanos» (support score); «determinar qué individuos responden a qué solicitaciones persuasivas» (responsiveness score) $)^{11}$.

De ahí que la inteligencia artificial, a través de la herramienta de los macrodatos, se preste a convertirse en un instrumento mediante el cual incidir en la democracia representativa, porque permite canalizar instancias populistas mediante actividades de microtargeting político, «es decir, un tipo de comunicación capaz de alcanzar persona por persona a cada uno de los electores de manera sustancialmente oculta, ya es una realidad. Los líderes carismáticos que interpretan el espíritu del pueblo y dialogan con él en mítines multitudinarios son reemplazados por líderes carismáticos digitales. Sociedades más o menos anónimas conjugan capacidad de gestionar macrodatos y perfiles individuales, orientando el voto, o al menos esa cantidad de votos suficiente para ser determinante y ganar las elecciones» ${ }^{12}$.

Se hace esencial, pues, identificar límites jurídicos adecuados para impedir que el uso de los macrodatos, y más en general el ejercicio de los poderes digitales de la Red, asuma un cariz tal que determine el fin de la democracia representativa, en nombre de una democracia digital directa, continua, inmediata, en la cual la persuasión digital termina por ocupar el lugar de la dialéctica democrática fundada en la confrontación entre opiniones distintas. Esto sin considerar el uso de herramientas de inteligencia artificial en regímenes autoritarios, lo que sin embargo puede conducir a una mayor compresión de las libertades políticas e individuales.

9 Nix, il cervello della campagna elettorale di Trump: "Grazie ai big data sappiamo cosa vogliono $i$ cittadini”, en La Stampa, 8 septiembre 2016.

10 R. DE Rosa, L'uso dei big data nella comunicazione politico-elettorale, en Comunicazione politica, 2/2018, p. 201 s.

11 R. De Rosa, trabajo citado, p. 201-202.

12 P. Ciarlo, Democrazia, partecipazione popolare e populismo al tempo della Rete, en Rivista AIC, 2/2018, p. 10. 
"Data drives all that we do», era el lema incluido en el sitio de Cambridge Analytica. A fin de que el uso de los macrodatos no guíe también la democracia, es necesario construir una ética de los datos que marque sus límites, sustituyendo a la materialidad del poder digital la abstracción de la regla de todos: son los principios del constitucionalismo ${ }^{13}$ los que nos guían en esta investigación.

\section{Title:}

Big data governance and representative democracy

\section{Summary:}

1. Big data and democracy. -2 . The multi-functionality of big data. -3 . Big data, populismo and representative democracy.

\section{Resumen:}

El fenómeno de los macrodatos tiene un impacto significativo en el ámbito político-institucional y en los canales tradicionales de representación política. La última elección presidencial de los Estados Unidos es una prueba clara. La campaña electoral que permitió a Trump llegar a la Presidencia es solo el último ejemplo que muestra cómo el uso de los datos está cambiando la relación entre ciudadanos e instituciones. Sin embargo, sería erróneo pensar que el fenómeno mencionado se limita a la realidad de los Estados Unidos. Los macrodatos, como se ha destacado en la literatura, difieren de los datos tradicionales (o datos pequeños) para tres características principales, las llamadas $3 \mathrm{~V}$ : volumen, velocidad y variedad. El impacto en el lado de las instituciones políticas es considerable, y está determinado sobre todo por la fuerte capacidad predictiva como característica típica de la utilización de los macrodatos. El procesamiento de una gran cantidad de datos termina en una imagen cercana y confiable de lo que sucederá, donde los pequeños datos más tradicionales siempre se han caracterizado por la inexactitud y el desorden. Esto es lo que se aplica a la minería de datos (es decir, el conjunto de técnicas y metodologías destinadas a extraer información de grandes cantidades de datos). En una fase en la que el circuito de representación política parece estar afectado por una crisis irreversible, los macrodatos corren el riesgo de transfigurar la relación entre ciudadanos e instituciones y merece un análisis profundo.

13 Compara M. Dogliani, Introduzione al diritto costituzionale, il Mulino, Bologna, 1994, p. 344. 


\begin{abstract}
:
The "big data" phenomenon turns out to have a significant impact on the political-institutional sphere and on traditional channels of political representation. The latest U.S. presidential election is a clear proof. The electoral campaign which allowed Trump to get to the Presidency is just the last example showing how the use of data is changing the relationship between citizens and institutions. It would be wrong, however, thinking that the mentioned phenomenon is limited to the U.S. reality. Big data, as has been highlighted in the literature, differ from traditional data (or small data) for three main characteristics, the so-called $3 \mathrm{Vs}$ : volume, velocity and variety. The impact on the political institutions side is considerable, and is determined above all by the strong predictive capacity as a typical feature of big data utilization. Processing large amount of data, ends up in a close and reliable picture of what is going to happen, where the more traditional small data have always been characterized by inaccuracy and disorder. This is what data mining (i.e. the set of techniques and methodologies aimed at extracting information from large amounts of data) apply for. In a phase where the political representation circuit seems to be afflicted by an irreversible crisis, big data risks to transfigure the relationship between citizens and institutions and it is worth of a deep analysis.
\end{abstract}

Palabras clave:

Macrodatos; democracia representativa; populismo.

Key words:

Big data; representative democracy; populism. 